\title{
Three-Dimensional (3D) Imaging with Infrared Matrix-Assisted Laser Desorption Electrospray Ionization (IR-MALDESI) Mass Spectrometry
}

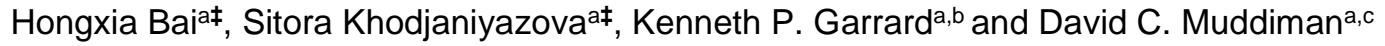 \\ ${ }^{a}$ FTMS Laboratory for Human Health Research, Department of Chemistry; ${ }^{b}$ Precision Engineering \\ Consortium; 'Molecular Education, Technology, and Research Innovation Center (METRIC), North \\ Carolina State University, Raleigh, NC 27695 USA \\ ${ }^{\ddagger}$ Authors contributed equally to this work
}

Submitted to: Journal of the American Society for Mass Spectrometry

Submitted: August 2, 2019

Revised: October 1, 2019

Supplemental materials: 4 pages, 2 Supplemental Tables / 3 Supplemental Figures

Keywords: Mass spectrometry imaging, 3D, IR-MALDESI, MUPS, HRAM

\author{
${ }^{*}$ Author for Correspondence \\ David C. Muddiman, Ph.D. \\ FTMS Laboratory for Human Health Research, Department of Chemistry and \\ Molecular Education, Technology, and Research Innovation Center (METRIC) \\ North Carolina State University \\ Raleigh, North Carolina 27695 \\ Phone: 919-513-0084 \\ Email: $\underline{\text { dcmuddim@ncsu.edu }}$
}




\section{SUPPLEMENTAL FIGURES}

Supplemental Table 1. Parameters for IR-MALDESI ionization source.

\begin{tabular}{c|c} 
x-stage used & Newport GTS-70 \\
\hline x-stage typical accuracy & $\pm 0.3 \mu \mathrm{m}$ \\
\hline x-stage guaranteed accuracy & $\pm 1.0 \mu \mathrm{m}$ \\
\hline y-stage used & Newport LTA-HS \\
\hline y-stage typical accuracy & $\pm 2.2 \mu \mathrm{m}$ \\
\hline y-stage guaranteed accuracy & $\pm 5.0 \mu \mathrm{m}$ \\
\hline z-stage used & $\pm 3.0 \mu \mathrm{m}$ \\
\hline z-stage accuracy & $6 \mathrm{~mm}$ \\
\hline inlet-to-stage distance & $5 \mathrm{~mm}$ \\
\hline laser-to-inlet distance & $1 \mathrm{~mm}$
\end{tabular}

Supplemental Table 2. Laser energies and Q-switch delays for depth resolution investigation.

\begin{tabular}{c|c|c|c}
$\#$ & $\begin{array}{c}\text { Q-Switch } \\
\text { Delay }(\mu \mathrm{s})\end{array}$ & $\begin{array}{c}\text { Arbitrary } \\
\text { Energy }(\%)\end{array}$ & $\begin{array}{c}\text { Energy at Target } \\
(\mathrm{mJ} \text { per pulse })\end{array}$ \\
\hline 1 & 680 & 100 & 1.20 \\
\hline 2 & 690 & 89 & 1.05 \\
\hline 3 & 700 & 78 & 1.00 \\
\hline 4 & 710 & 67 & 0.90 \\
\hline 5 & 720 & 56 & 0.70 \\
\hline 6 & 730 & 44 & 0.50 \\
\hline 7 & 740 & 33 & 0.30 \\
\hline 8 & 750 & 22 & 0.10 (not stable) \\
\hline 9 & 760 & 11 & could not detect \\
\hline 10 & 770 & 0 & could not detect
\end{tabular}


a)

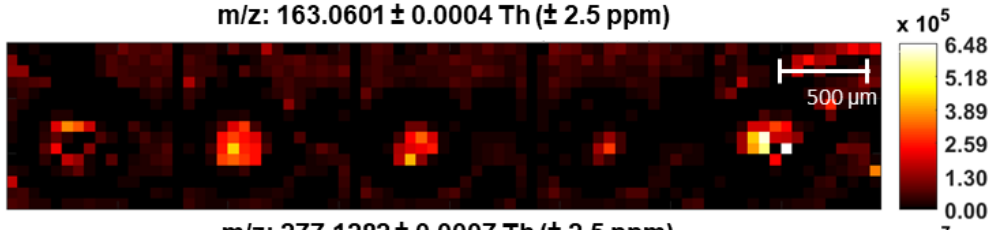

b)

$\mathrm{m} / \mathrm{z}: 277.1282 \pm 0.0007 \mathrm{Th}( \pm 2.5 \mathrm{ppm})$

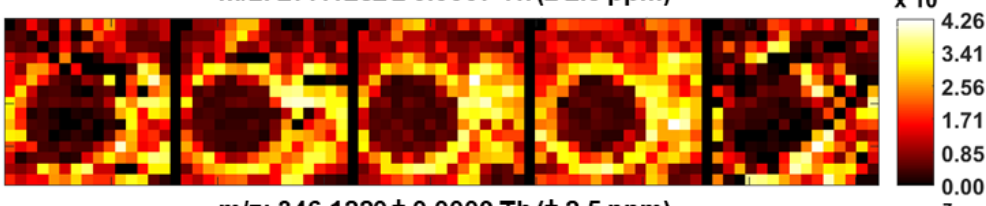

c)

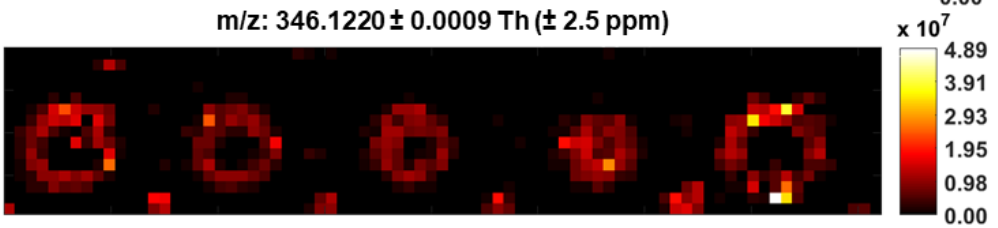

d)

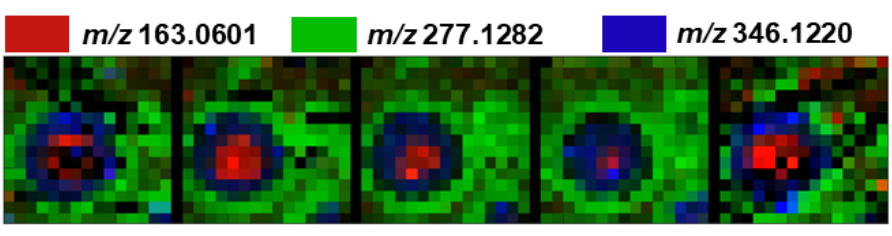

Supplemental Figure 1. Ion heatmaps of A peak representing three markers: a) starch, b) triethyl citrate, c) omeprazole and d) their colocalization across 5 layers in the pill trimmed in half. 


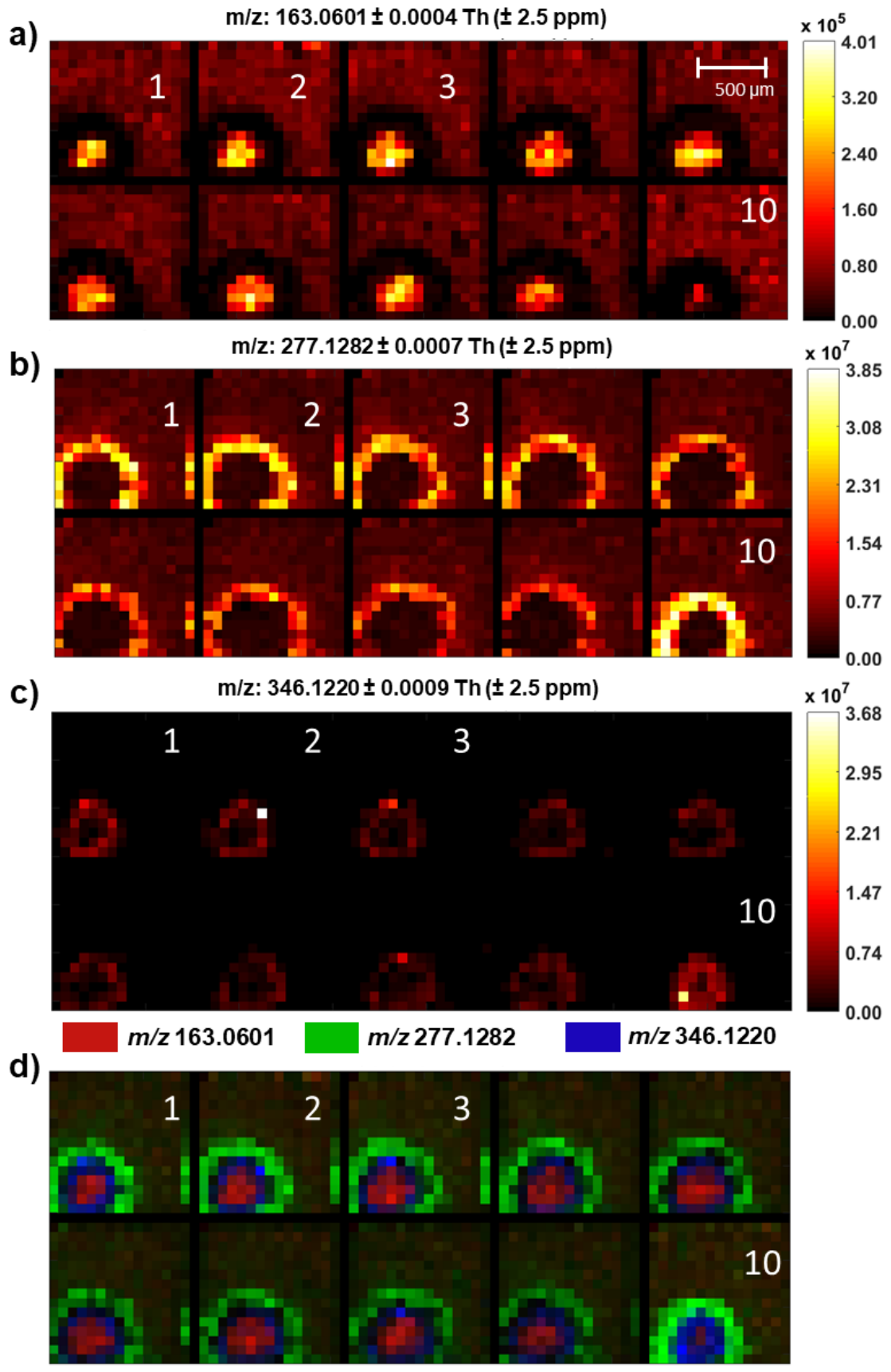

Supplemental Figure 2. Ion heatmaps of A peak representing three markers: a) starch, b) triethyl citrate, c) omeprazole and d) their colocalization across 10 layers in the pill trimmed in half. 


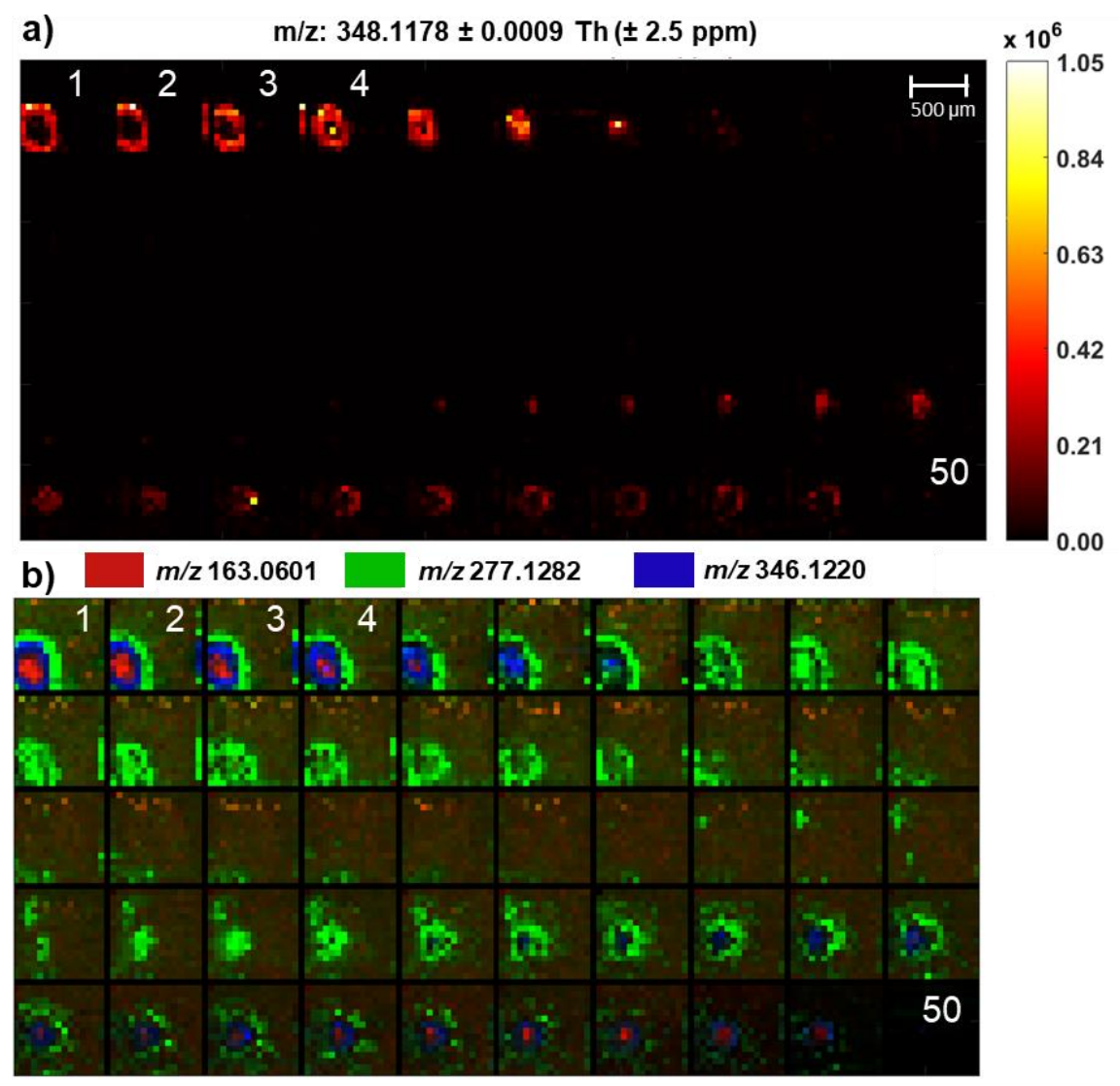

Supplemental Figure 3. a) The distribution of omeprazole's ${ }^{34} S_{1}$ peak ( $\left.m / z 348.1178\right)$ on the half pill; b) ion colocalization of A peaks of starch $(\mathrm{m} / \mathrm{z} 163.0601)$, triethyl citrate $(\mathrm{m} / \mathrm{z} 277.1282)$ and omeprazole $(\mathrm{m} / \mathrm{z}$ 346.1220) across 50 layers. 